\title{
Epidemiology, causes, evolution and outcome in a single-center cohort of 1116 critically ill patients with hypoxic hepatitis
}

\author{
Astrid Van den broecke ${ }^{1,3^{*}+}$ D , Laura Van Coile ${ }^{1 \dagger}$, Alexander Decruyenaere ${ }^{1,2}$, Kirsten Colpaert $^{1,3}$, \\ Dominique Benoit ${ }^{1,3}$, Hans Van Vlierberghe ${ }^{1,4}$ and Johan Decruyenaere ${ }^{1,3}$
}

\begin{abstract}
Background: Hypoxic hepatitis $(\mathrm{HH})$ is a type of acute hepatic injury that is histologically characterized by centrilobular liver cell necrosis and that is caused by insufficient oxygen delivery to the hepatocytes. Typical for HH is the sudden and significant increase of aspartate aminotransferase (AST) in response to cardiac, circulatory or respiratory failure. The aim of this study is to investigate its epidemiology, causes, evolution and outcome.
\end{abstract}

Methods: The screened population consisted of all adults admitted to the intensive care unit (ICU) at the Ghent University Hospital between January 1, 2007 and September 21, 2015. HH was defined as peak AST > 5 times the upper limit of normal (ULN) after exclusion of other causes of liver injury. Thirty-five variables were retrospectively collected and used in descriptive analysis, time series plots and Kaplan-Meier survival curves with multi-group log-rank tests.

Results: $\mathrm{HH}$ was observed in $4.0 \%$ of the ICU admissions at our center. The study cohort comprised 1116 patients. Causes of HH were cardiac failure (49.1\%), septic shock (29.8\%), hypovolemic shock (9.4\%), acute respiratory failure (6.4\%), acute on chronic respiratory failure (3.3\%), pulmonary embolism (1.4\%) and hyperthermia (0.5\%). The 28-day mortality associated with $\mathrm{HH}$ was $45.0 \%$. Mortality rates differed significantly $(P=0.007)$ among the causes, ranging from $33.3 \%$ in the hyperthermia subgroup to 52.9 and $56.2 \%$ in the septic shock and pulmonary embolism subgroups, respectively. The magnitude of AST increase was also significantly correlated $(P<0.001)$ with mortality: 33.2, 44.4 and $55.4 \%$ for peak AST 5-10x ULN, 10-20x ULN and > 20x ULN, respectively.

Conclusion: This study surpasses by far the largest cohort of critically ill patients with $\mathrm{HH}$. $\mathrm{HH}$ is more common than previously thought with an ICU incidence of $4.0 \%$, and it is associated with a high all-cause mortality of $45.0 \%$ at 28 days. The main causes of $\mathrm{HH}$ are cardiac failure and septic shock, which include more than 3/4 of all episodes. Clinicians should search actively for any underlying hemodynamic or respiratory instability even in patients with moderately increased AST levels.

Keywords: Critical care medicine, Critically ill, Epidemiology, Hypoxic hepatitis, Intensive care medicine, Ischemic hepatitis, Liver failure, Mortality, Outcome, Shock liver

\section{Background}

Hypoxic hepatitis $(\mathrm{HH})$, also referred to as "ischemic hepatitis" or "shock liver," is a type of acute hepatic injury

\footnotetext{
*Correspondence: Astrid.Vandenbroecke@UGent.be

${ }^{\dagger}$ Astrid Van den broecke and Laura Van Coile have contributed equally to this study and are the joint first authors

${ }^{3}$ Department of Intensive Care Medicine, Ghent University Hospital, De Pintelaan 185, 9000 Ghent, Belgium

Full list of author information is available at the end of the article
}

that is histologically characterized by centrilobular liver cell necrosis and that is caused by insufficient oxygen delivery to the hepatocytes [1]. Typical for this form of liver cell necrosis is the sudden and significant increase of aspartate aminotransferase (AST) in response to cardiac, circulatory or respiratory failure [1]. Although often missed, $\mathrm{HH}$ is a fairly common cause of hepatic dysfunction in an intensive care unit (ICU) with a pooled incidence of $2.5 \%$ from a recent meta-analysis of 1782 
patients [2]. Its incidence varies widely among published studies, ranging from 0.16 to $12 \%$, depending upon institution, population studied and definition used [1-8]. However, a high associated mortality of approximately $50 \%$ has been consistently observed in all studies [2-6, 8].

The major causes of $\mathrm{HH}$ are septic shock, respiratory failure and cardiogenic shock [1, 2]. Possible pathophysiological mechanisms include (1) ischemia due to reduced blood supply (forward failure) or due to right heart failure (backward failure) with venous congestion, (2) hypoxemia due to reduced blood oxygenation and (3) increased oxygen consumption due to elevated metabolic demand (e.g., in severe hyperthermia or septic shock) [1, 9]. Patients with comorbidities are more likely to develop $\mathrm{HH}$, as they have an increased vulnerability even to minor hemodynamic or respiratory insults, such as short periods of hypotension or hypoxemia. These comorbidities contribute substantially to the high mortality associated with $\mathrm{HH}[4,7,8]$.

$\mathrm{HH}$ is reflected by a typical pattern of liver enzyme alterations. It presents with a sudden and significant increase of AST, alanine aminotransferase (ALT) and lactate dehydrogenase (LDH), reaching their peak levels around $24 \mathrm{~h}$ after ICU admission [4] and declining steadily to baseline within 10-15 days [1]. Initially, AST exceeds ALT, but as ALT declines more slowly, a reversal of the AST/ALT ratio is observed within 3 days after the peak $[5,6,8,10]$. Although this biochemical pattern is highly suggestive of $\mathrm{HH}$, it is not pathognomonic and warrants further evaluation [1]. Other common causes of significant increases in aminotransferase levels are druginduced liver injury (e.g., acetaminophen toxicity) and acute viral hepatitis. However, studies have shown that a sudden and significant increase of AST is caused by $\mathrm{HH}$ in more than $50 \%$ of the cases $[11,12]$. Furthermore, $\mathrm{HH}$ is frequently associated with a prolonged prothrombin time and accompanied by additional evidence of endorgan hypoperfusion, such as impaired renal function and increased lactate level [3-6]. A rapid rise and subsequent fall in aminotransferase levels with reversal of the initial AST/ALT ratio, a prolongation of prothrombin time and an increase in serum creatinine level comprise a triad of biochemical abnormalities that can suggest the diagnosis of $\mathrm{HH}$, as proposed by Raurich et al. [6].

Currently, only limited data from small retrospective studies are available, making $\mathrm{HH}$ an understudied disease. The largest cohort described to date was recently published and included 565 patients [8]. The aim of this study is to investigate in detail the epidemiology, causes, evolution and outcome of $\mathrm{HH}$ in a large single-center cohort. More insight in $\mathrm{HH}$ may improve awareness and facilitate earlier diagnosis.

\section{Methods}

\section{Study cohort and data collection}

The screened population consisted of all consecutive adults ( $\geq 18$ years, $n=29,874$ ) who were admitted to the surgical, cardiac or medical ICU at the Ghent University Hospital between January 1, 2007 and September 21,2015 . HH was defined as a significant but transient increase in AST level above 5 times the upper limit of normal (ULN) after exclusion of other potential causes of liver injury. Different AST cutoff values for defining $\mathrm{HH}$ are used in the literature [2]. As $\mathrm{HH}$ has been histologically proven to occur even in patients with moderately elevated AST levels (at AST levels of 252 and $300 \mathrm{IU} / \mathrm{L}$ in the cohort of Cohen et al. [13] and Bynum et al. [14], respectively), a cutoff of at least 5 times the ULN was used in this study, i.e., 155 and $185 \mathrm{U} / \mathrm{L}$ for females and males, respectively. The 4012 identified patients whose AST level exceeded our cutoff value were evaluated for the presence of $\mathrm{HH}$ by three independent experts based on the pattern of liver enzyme alterations, the daily clinical notes and the discharge summaries. The sine qua non was the exposure to a hemodynamic or respiratory insult preceding the AST increase and the exclusion of other potential causes of liver injury. A flow diagram of the exclusion criteria is shown in Fig. 1. Reasons for exclusion were (1) acute liver failure, (2) chronic liver failure, (3) other conditions associated with abnormal liver tests such as cholangitis and pancreatitis, (4) liver surgery, (5) surgery near the liver, (6) hepatic vessel injury or thrombosis, (7) rhabdomyolysis, (8) an unclear increase of creatine kinase (CK), (9) post-anesthesia without overt evidence of an acute cardiac or respiratory event perioperatively, (10) missing data and (11) duplicate patients. (In patients having developed multiple episodes of $\mathrm{HH}$ during the study period, only the first episode is eligible for analysis.) Rhabdomyolysis was defined as serum CK exceeding 5 times the ULN (i.e., 850 and $974 \mathrm{U} / \mathrm{L}$ for females and males, respectively) with a CK-MB/CK ratio below 6\% [6]. Patients with elevated CK levels but with unknown CK-MB value were excluded due to the uncertain CK increase. This study was approved by the Ethics Committee of the Ghent University Hospital (project numbers 2015/0796-0797). Due to the retrospective nature of this study, the need for informed consent was waived.

Routinely available biochemical parameters were recorded, including AST (U/L), ALT (U/L), LDH (U/L), bilirubin (mg/dL), alkaline phosphatase (AP) (U/L), gamma-glutamyl transpeptidase (U/L), lipase (U/L), international normalized ratio (INR), platelet count $\left(10^{3} /\right.$ $\mu \mathrm{L})$, white blood cell count $\left(10^{3} / \mu \mathrm{L}\right)$, hemoglobin $(\mathrm{g} / \mathrm{dL})$, CK $(\mathrm{U} / \mathrm{L})$, creatinine $(\mathrm{mg} / \mathrm{dL})$, urea $(\mathrm{mg} / \mathrm{dL})$ and lactate $(\mathrm{mg} / \mathrm{dL})$. Values at specific time points were estimated 


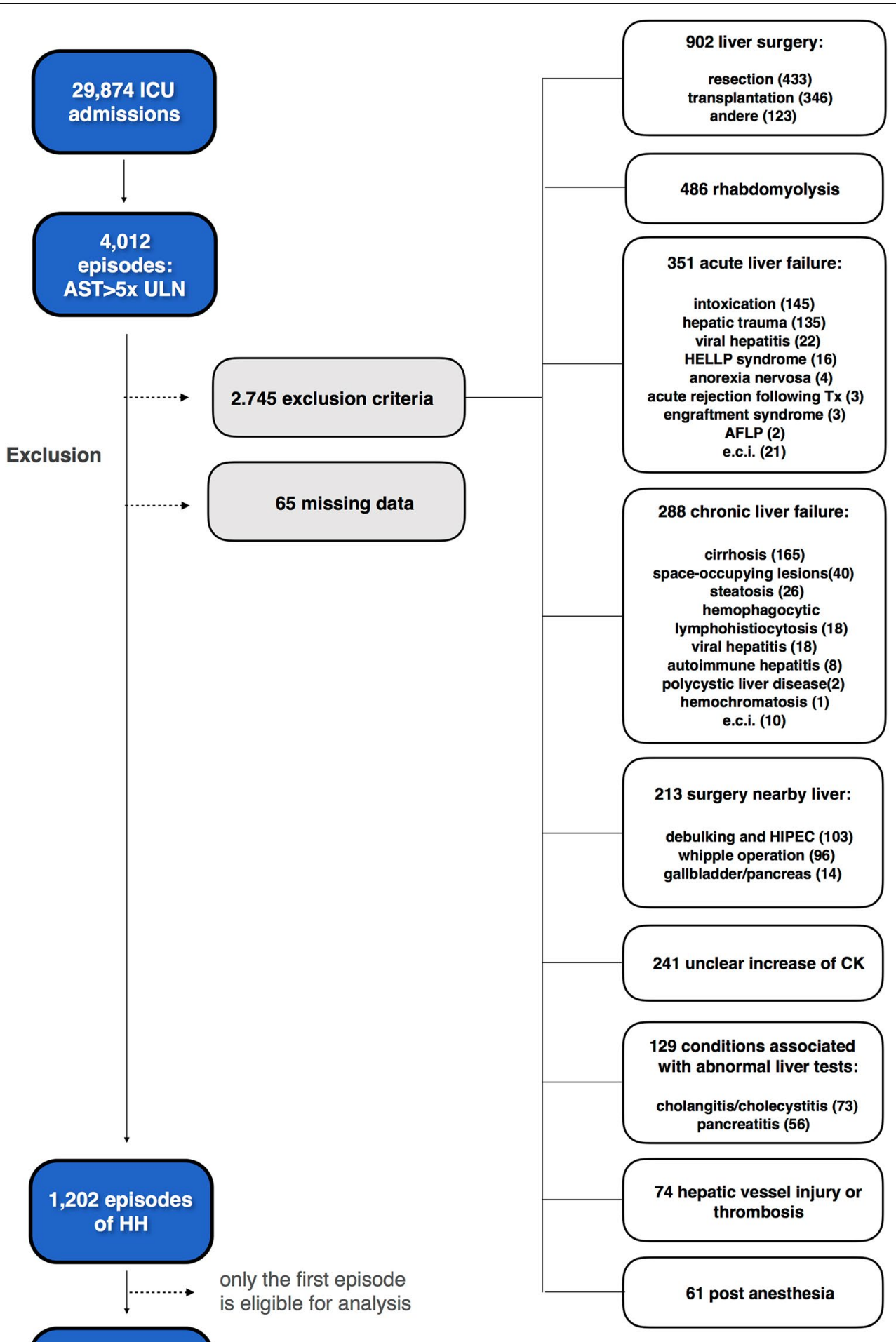

1,116 unique

episodes of $\mathrm{HH}$

Fig. 1 Flow diagram of the exclusion criteria. AFLP acute fatty liver of pregnancy, AST aspartate aminotransferase, CK creatine kinase, e.c.i e causa ignota, HELLP hemolysis, elevated liver enzymes and low platelet count, HH hypoxic hepatitis, HIPEC hyperthermic intraperitioneal chemotherapy, ICU intensive care unit 
using linear interpolation between consecutive recorded values. Parameters related to patient characteristics included sex, age, body mass index (BMI) and comorbidities (diabetes mellitus, cardiac function and chronic respiratory disease). Parameters related to the episode of $\mathrm{HH}$ included cause, severity of illness scores [acute physiology II score (APS-II) and the simplified acute physiology II score (SAPS-II)], supportive therapy (inotropic agents, vasopressor agents, mechanical ventilation, intra-aortic balloon pump (IABP) and need for dialysis), ICU and hospital length of stay, duration of the HH episode, and ICU and in-hospital mortality at 28 days. The peak of $\mathrm{HH}$ was defined as the point in time (T-ASTmax) when AST reached its peak value (ASTmax). In this study, the time origin was set to T-ASTmax (designated as time 0 ) and all recorded values are expressed in time relative to T-ASTmax for optimal comparison. Severity of illness scores were recorded over a time span of $24 \mathrm{~h}$ around T-ASTmax. Three categories for severity of $\mathrm{HH}$

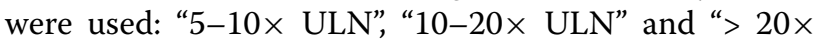
ULN". Seven underlying causes of $\mathrm{HH}$ were defined: cardiac failure, septic shock, hypovolemic shock, pulmonary embolism, acute respiratory failure, acute on chronic respiratory failure and hyperthermia.

\section{Statistical analysis}

Categorical data are reported as counts and percentages. Continuous data are reported as the median with the first (Q1) and third (Q3) quartiles. For categorical variables, comparisons between groups are performed using the Pearson's Chi square test for contingency. For continuous variables, a permutation test based on difference in medians between groups is used. A Tukey-like approach with permutation resampling is applied to adjust $P$ values for multiple pairwise comparisons. All statistical tests are performed as two-sided tests at a significance level of 0.05 . The time trend of laboratory variables is graphically assessed using time series plots and stacked bar charts. Kaplan-Meier survival curves until 28 days after T-ASTmax and multi-group log-rank tests are used to compare the all-cause mortality between groups. Statistical analysis is performed using $\mathrm{R}$ version 3.3.2 [15].

\section{Results}

\section{Patient and episode characteristics}

During the study period, 29,874 adult patients with a male-to-female ratio of approximately $3: 2$ were admitted to the ICU at our center, of whom 4012 patients had peak AST levels exceeding 5 times the ULN. In 30.0\% $(1202 / 4012)$ of the cases, the elevation of AST was caused by $\mathrm{HH}$, resulting in an overall ICU incidence of $4.0 \%(1202 / 29,874)$. As only the first episode was eligible for analysis in patients with multiple episodes of $\mathrm{HH}$ during the study period, the final study cohort comprised 1116 patients.

The all-cause mortality of patients admitted to the ICU at our center during the study period was $7.7 \%$ $(2302 / 29,874)$. Among these non-survivors, $19.8 \%$ (455/2302) had developed HH during their ICU stay. The all-cause mortality associated with $\mathrm{HH}$ was $45.0 \%$ $(502 / 1116)$ at 28 days, of which $90.6 \%$ occurred during ICU stay. The survival curves by cause of $\mathrm{HH}$ are presented in Fig. 2.

In our study cohort, the median age was 66.0 (Q1-Q3 55.0-74.0) years with a male-to-female ratio of 3:2. The median SAPS-II score at T-ASTmax was 66.0 (Q1-Q3

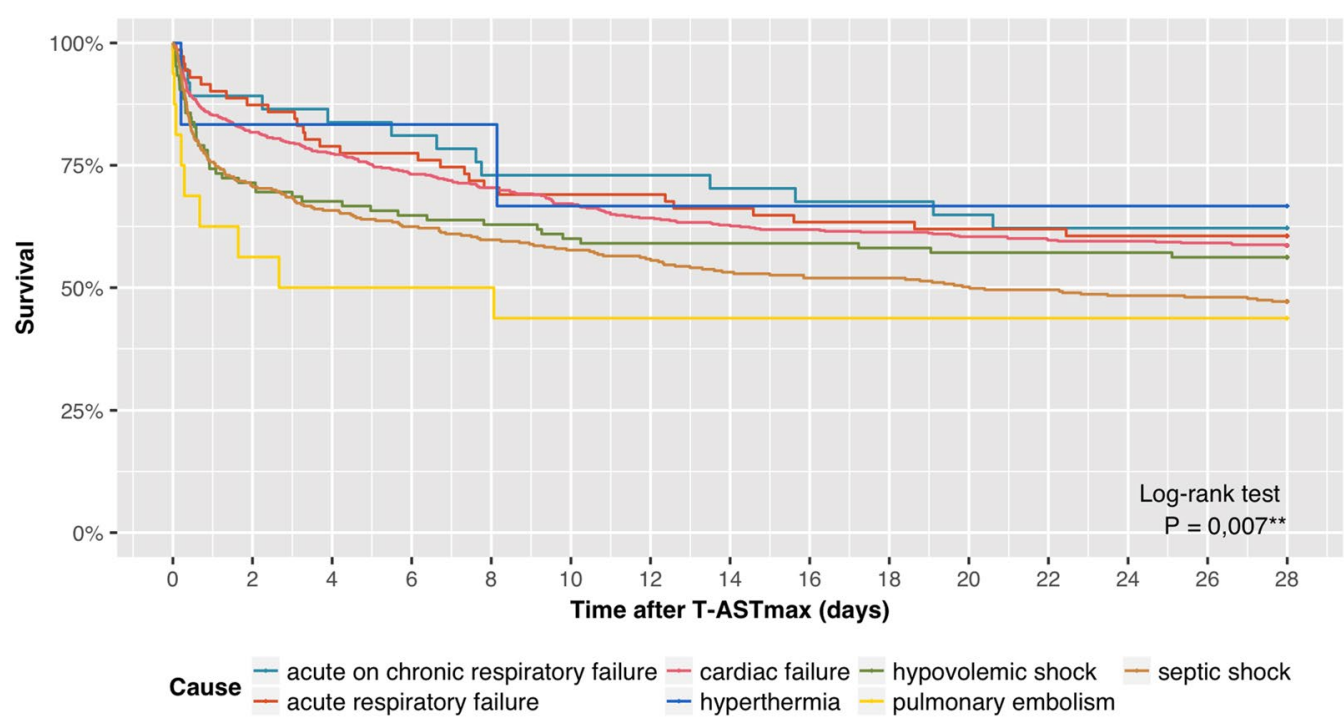

Fig. 2 Survival curves by cause of hypoxic hepatitis. T-ASTmax is designated as the time 0. T-ASTmax time point of maximum AST value 
43.0-81.0). $74.9 \%$ of patients required mechanical ventilation. Vasopressor and inotropic agents were used in 60.8 and $43.2 \%$, respectively, and $9.5 \%$ were on dialysis at T-ASTmax. The causes of $\mathrm{HH}$, in decreasing order of frequency, were cardiac failure (49.1\%), septic shock (29.8\%), hypovolemic shock (9.4\%), acute respiratory failure $(6.4 \%)$, acute on chronic respiratory failure $(3.3 \%)$, pulmonary embolism (1.4\%) and hyperthermia (0.5\%). An episode of $\mathrm{HH}$ (length of time that AST levels are exceeding 5 times the ULN) had a median duration of 54.3 (Q1-Q3 26.4-94.2) h, during which the median recovery time (duration from peak AST to levels below 5 times the ULN) was 34.7 (Q1-Q3 14.6-67.2) h.

Survivors had significantly lower severity of illness scores (median SAPS-II score of 54.0 vs. 76.0, $P<0.001$ and median APS-II score 25.0 vs. 30.0, $P=0.005$ ) as compared to non-survivors. They were less likely to have septic shock (25.6 vs. $35.1 \%, P=0.003$ ). Peak AST levels above 20 times ULN were less commonly seen in survivors (33.4 vs. $50.8 \%, P<0.001)$. More characteristics, classified according to survival status, are presented in Table 1.

The three ASTmax subgroups ("5-10× ULN" vs. "10$20 \times$ ULN" vs. "> $20 \times$ ULN") were also compared with each other. The causes of $\mathrm{HH}$ were equally represented within each subgroup. Patients with higher peak AST levels had higher severity of illness scores (median SAPSII score of 57.0 vs. 60.0 vs. 73.0, $P<0.001$; median APSII score of 24.0 vs. 26.5 vs. $32.0, P<0.001$ ) and a higher need for supportive therapy, such as inotropic agents (38.5 vs. 37.9 vs. $50.2 \%, P<0.001$ ), vasopressor agents (50.9 vs. 59.4 vs. $70.0 \%, P<0.001$ ), dialysis (4.8 vs. 8.4 vs. $14.1 \%, P<0.001)$ and IABP $(13.7$ vs. $20.7 \%$ vs. $18.9 \%$, $P=0.041)$. In addition, higher peak AST levels were associated with higher 28-day mortality rates (33.2 vs. 44.4 vs. $55.4 \%, P<0.001)$. More characteristics, classified according to severity of $\mathrm{HH}$ and to cause of $\mathrm{HH}$, are presented in Tables 2 and 3, respectively.

\section{Trend of liver tests}

The time trend of the liver tests is illustrated in Fig. 3. Once the AST level exceeded 5 times the ULN (designated as start of $\mathrm{HH}$ ), it reached a median peak level of 521.2 (Q1-Q3 269.1-1581.6) U/L within a median of 16.3 (Q1-Q3 7.9-26.8) h. Subsequently, AST started to decline, dropping below 5 times the ULN after a median time of 34.7 (Q1-Q3 14.6-67.2) h and normalizing after a median time of 6.4 (Q1-Q3 3.9-10.9) days in survivors.

ALT and LDH peaked at median values of 332.0 (Q1-Q3 149.4-953.6) U/L and 1180.6 (Q1-Q3 684.12770.8) U/L within a median time of 22.0 (Q1-Q3 9.743.0) and 21.4 (Q1-Q3 10.0-43.6) h after start of $\mathrm{HH}$, respectively. In survivors, $\mathrm{LDH}$ returned to baseline
(< $233 \mathrm{U} / \mathrm{L})$ within a median of 5.1 (Q1-Q3 1.3-15.9) days after its peak. ALT declined more slowly, returning to baseline $(<31$ and $<40 \mathrm{U} / \mathrm{L}$ for females and males, respectively) within 8.6 (Q1-Q3 3.9-16.5) days after its peak. When considering all patients, including those with AST already exceeding 5 times the ULN at ICU admission, ALT and LDH peaked at T-ASTmax in the majority of patients (65.5 and 53.1\%, respectively) and to a lesser extent after T-ASTmax (26.2 and 29.8\%, respectively).

The median relative increase of AST, ALT and LDH at T-ASTmax was 15.0, 8.7 and 4.7 times their ULN, respectively. LDH exceeded both AST and ALT at all time points in more than $80 \%$ of patients. The median AST/ ALT ratio was 1.6 at the start of $\mathrm{HH}$, increased to 1.8 at T-ASTmax and subsequently declined, reversing within a median of $32.5 \mathrm{~h}$ after T-ASTmax.

In addition, Fig. 3 illustrates that the median INR, creatinine and bilirubin levels also peaked at T-ASTmax, albeit to a much less extent, with median bilirubin level never exceeding the ULN. This is also illustrated in Fig. 4. Both in survivors and non-survivors, the proportion of patients with bilirubin level above the ULN increased mildly at T-ASTmax and then gradually decreased again. The proportion of survivors with bilirubin above 3 times the ULN was limited and increased slightly later during ICU stay. Likewise, AP remained stable during $\mathrm{HH}$, except for a mild increase later during ICU stay, as illustrated in Figs. 3 and 4.

The pattern of liver enzyme alterations described above was found in all ASTmax subgroups (see Appendix Figs. 5, 6, 7). Median values of other laboratory tests at start of $\mathrm{HH}$ and at T-ASTmax are presented in Table 4.

\section{8-day mortality}

$\mathrm{HH}$ was associated with high mortality rates, especially early in its course. $18.5 \%$ died within $24 \mathrm{~h}$ after T-ASTmax. After this first day, the hazard declined dramatically, but remained quite high, resulting in 40.2 and $45.0 \%$ all-cause mortality at 14 and 28 days, respectively. $90.6 \%$ of deaths occurred during ICU stay.

Twenty-eight-day mortality rates differed significantly among the causes of $\mathrm{HH}(P=0.007)$. The high number of early deaths in the pulmonary embolism, septic shock and hypovolemic shock subgroups mainly accounted for the early mortality associated with HH. 37.5, 24.3, 25.7\% of these patients died within $24 \mathrm{~h}$ after T-ASTmax. The mortality rate in the pulmonary embolism subgroup exceeded at all times those of other subgroups and ultimately resulted in $56.2 \%$ mortality at 28 days. The septic shock and hypovolemic shock subgroups had a 28-day mortality of 52.9 and $43.8 \%$, respectively. While the early mortality in the cardiac failure subgroup was less pronounced, yet still considerable as $14.8 \%$ of these patients 


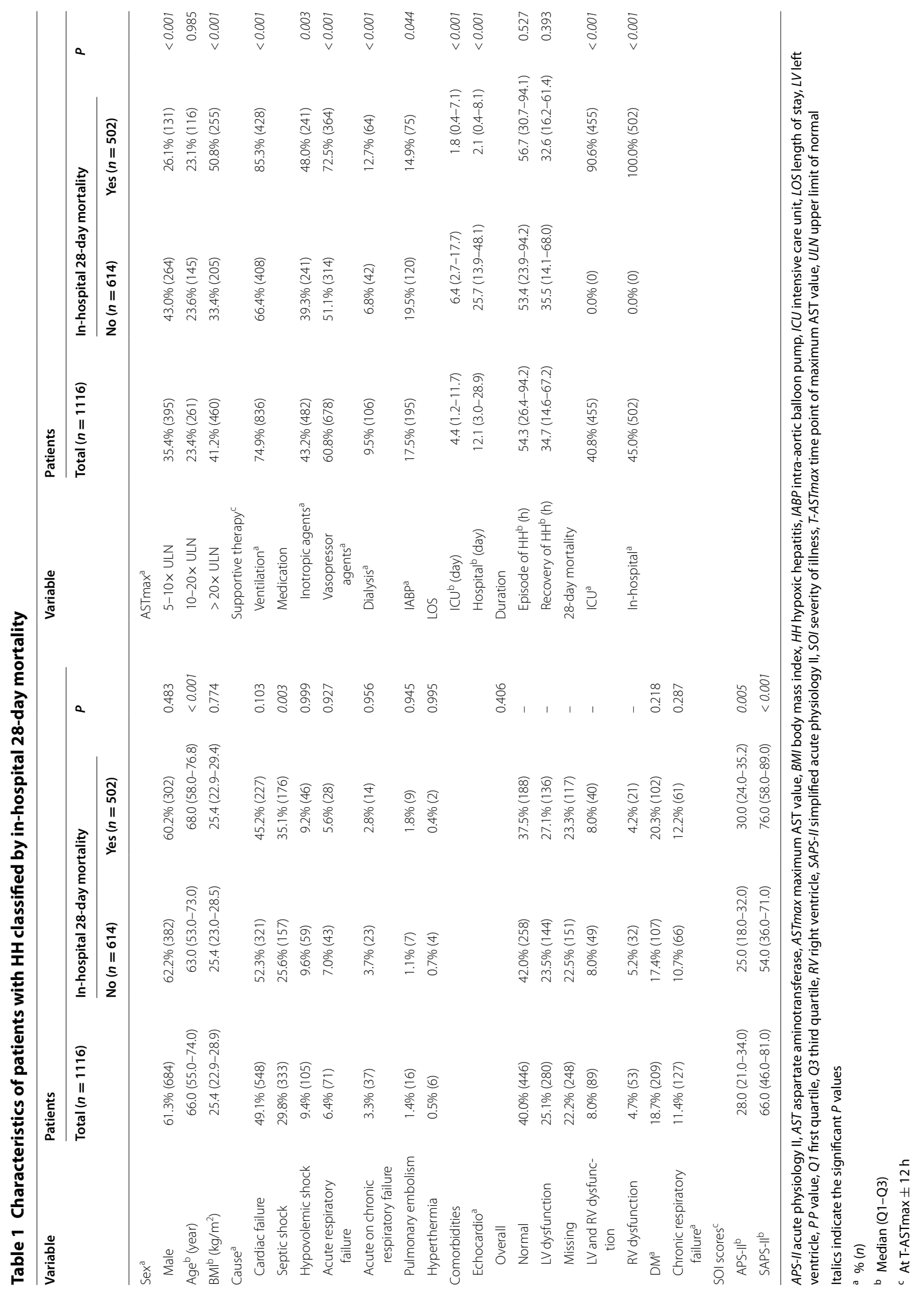




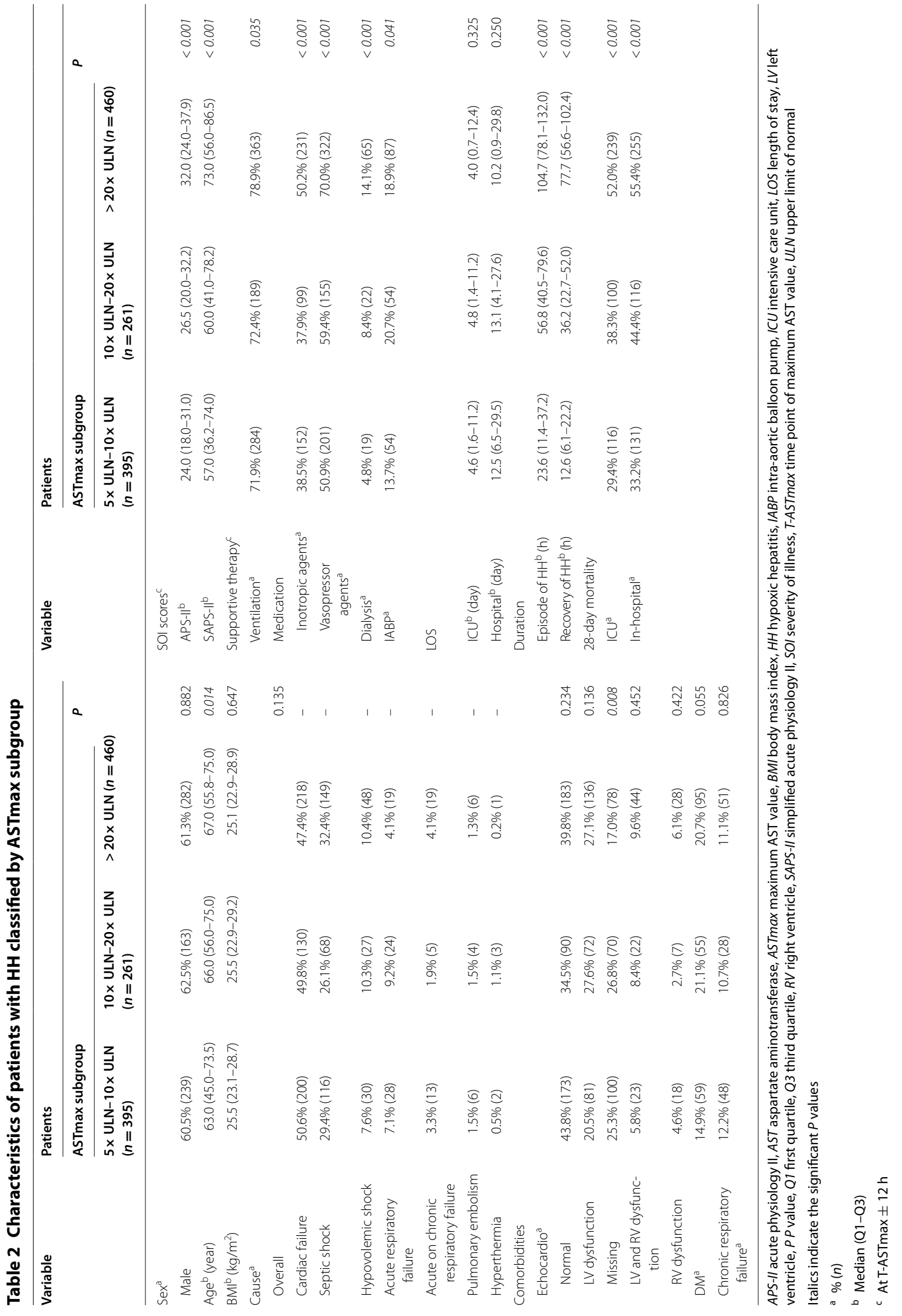


Table 3 Characteristics of patients with HH classified by clinical cause

\begin{tabular}{|c|c|c|c|c|c|c|c|c|}
\hline \multirow[t]{2}{*}{ Variable } & \multicolumn{8}{|l|}{ Cause } \\
\hline & $\begin{array}{l}\text { Cardiac failure } \\
(n=548)\end{array}$ & $\begin{array}{l}\text { Septic shock } \\
(n=333)\end{array}$ & $\begin{array}{l}\text { Hypov- } \\
\text { olemic shock } \\
(n=105)\end{array}$ & $\begin{array}{l}\text { Acute res- } \\
\text { piratory failure } \\
(n=71)\end{array}$ & $\begin{array}{l}\text { Acute } \\
\text { on chronic res- } \\
\text { piratory failure } \\
(n=37)\end{array}$ & $\begin{array}{l}\text { Pulmonary } \\
\text { embolism } \\
(n=16)\end{array}$ & $\begin{array}{l}\text { Hyperthermia } \\
(n=6)\end{array}$ & $P$ \\
\hline \multicolumn{9}{|l|}{ Sex ${ }^{a}$} \\
\hline Male & $63.1 \%(346)$ & $61.6(205)$ & $57.1 \%(60)$ & $54.9 \%(39)$ & $54.1 \%(20)$ & $62.5(10)$ & $66.7 \%(4)$ & 0.721 \\
\hline Age $^{b}$ (year) & $68.0(58.0-76.0)$ & $64.0(52.0-74.0)$ & $64.0(56.0-73.0)$ & $59.0(48.5-71.5)$ & $65.0(61.0-72.0)$ & $57.5(47.5-61.5)$ & $54.5(35.8-64.2)$ & 0.003 \\
\hline $\mathrm{BMI}^{\mathrm{b}}\left(\mathrm{kg} / \mathrm{m}^{2}\right)$ & $25.5(23.3-28.7)$ & $25.0(22.1-29.3)$ & $26.2(23.4-28.9)$ & $24.2(22.0-27.8)$ & $24.6(22.1-27.3)$ & $27.8(25.1-31.0)$ & $27.6(23.7-29.3)$ & 0.202 \\
\hline \multicolumn{9}{|l|}{ Comorbidities } \\
\hline \multicolumn{9}{|l|}{ Echocardio $^{a}$} \\
\hline Normal & $32.7 \%$ (179) & $51.1 \%(170)$ & $43.8 \%(46)$ & $36.6 \%(26)$ & $48.6 \%(18)$ & $18.8 \%(3)$ & $66.7 \%(4)$ & $<0.001$ \\
\hline LV dysf. & $36.5 \%(200)$ & $15.9 \%(53)$ & $5.7 \%(6)$ & $21.1 \%(15)$ & $10.8 \%(4)$ & $6.2 \%(1)$ & $16.7 \%(1)$ & $<0.001$ \\
\hline Missing & $13.3 \%(73)$ & $26.1 \%(87)$ & $46.7 \%(49)$ & $35.2 \%(25)$ & $24.3 \%(9)$ & $25.0 \%(4)$ & $16.7 \%(1)$ & $<0.001$ \\
\hline $\begin{array}{l}\text { LV and RV } \\
\text { dysf. }\end{array}$ & $13.0 \%(71)$ & $3.3 \%(11)$ & $1.0 \%(1)$ & $4.2 \%(3)$ & $2.7 \%(1)$ & $12.5 \%(2)$ & $0.0 \%(0)$ & $<0.001$ \\
\hline RV dysf. & $4.6 \%(25)$ & $3.6 \%(12)$ & $2.9 \%(3)$ & $2.8 \%(2)$ & $13.5 \%(5)$ & $37.5 \%(6)$ & $0.0 \%(0$ & $<0.001$ \\
\hline $\mathrm{DM}^{\mathrm{a}}$ & $20.8 \%(114)$ & $19.2 \%(64)$ & $10.5 \%(11)$ & $12.7 \%(9)$ & $24.3 \%(9)$ & $12.5 \%(2)$ & $0.0 \%(0)$ & 0.103 \\
\hline $\begin{array}{l}\text { Chronic } \\
\text { respiratory } \\
\text { failure }^{a}\end{array}$ & $8.4 \%(46)$ & $9.6 \%(32)$ & $9.5 \%(10)$ & $0.0 \%(\%)$ & $100.0 \%(37)$ & $12.5 \%(2)$ & $0.0 \%(0)$ & $<0.001$ \\
\hline \multicolumn{9}{|l|}{ SOl scores ${ }^{c}$} \\
\hline APS- $\|^{b}$ & $28.0(23.0-35.0)$ & $26.0(21.0-35.0)$ & $26.0(18.5-32.5)$ & $30.0(28.0-34.0)$ & $26.5(24.2-30.8)$ & $36.5(36.2-36.8)$ & $21.5(19.2-25.0)$ & 0.590 \\
\hline SAPS- \|\|$^{b}$ & $66.0(44.0-79.0)$ & $67.0(49.0-84.0)$ & $65.0(48.0-82.0)$ & $63.0(31.0-79.0)$ & $67.5(55.0-73.5)$ & $83.0(69.2-99.2)$ & $41.5(34.0-48.2)$ & 0.171 \\
\hline \multicolumn{9}{|l|}{ ASTmax ${ }^{a}$} \\
\hline Overall & & & & & & & & 0.135 \\
\hline $5-10 \times$ ULN & $36.5 \%(200)$ & $34.8 \%(116)$ & $28.6 \%(30)$ & $39.4 \%(28)$ & $35.1 \%(13)$ & $37.5 \%(6)$ & $33.3 \%(2)$ & - \\
\hline $10-20 \times$ ULN & $23.7 \%(130)$ & $20.4 \%(68)$ & $25.7 \%(27)$ & $33.8 \%(24)$ & $13.5 \%(5)$ & $25.0 \%(4)$ & $50.0 \%(3)$ & - \\
\hline$>20 \times$ ULN & $39.8 \%(218)$ & $44.7 \%$ (149) & $45.7 \%(48)$ & $26.8 \%(19)$ & $51.4 \%(19)$ & $37.5 \%(6)$ & $16.7 \%(1)$ & - \\
\hline \multicolumn{9}{|l|}{$\begin{array}{c}\text { Supportive } \\
\text { therapy }^{c}\end{array}$} \\
\hline Ventilation $^{a}$ & $79.2 \%(434)$ & $73.0 \%(243)$ & $70.5 \%(74)$ & $60.6 \%(43)$ & $64.9(24)$ & $75.0 \%(12)$ & $100.0 \%(6)$ & 0.004 \\
\hline \multicolumn{9}{|l|}{ Medication } \\
\hline $\begin{array}{l}\text { Inotropic } \\
\text { agents }^{\mathrm{a}}\end{array}$ & $63.1 \%(346)$ & $26.4 \%(88)$ & $21.0 \%(22)$ & $12.7 \%(9)$ & $21.6 \%(8)$ & $43.8 \%(7)$ & $33.3 \%(2)$ & $<0.001$ \\
\hline $\begin{array}{c}\text { Vasopressor } \\
\text { agents }^{\mathrm{a}}\end{array}$ & $58.6 \%(321)$ & $69.1 \%(230)^{d}$ & $61.9 \%(65)$ & $46.5 \%(33)$ & $43.2 \%(16)$ & $75.0 \%(12)$ & $16.7 \%(1)$ & $<0.001$ \\
\hline Dialysis $^{a}$ & $10.4 \%(57)$ & $11.1 \%(37)$ & $2.9 \%(3)$ & $9.9 \%(7)$ & $2.7 \%(1)$ & $0.0 \%(0)$ & $16.7 \%(1)$ & 0.090 \\
\hline $\mathrm{ABP} \mathrm{P}^{\mathrm{a}}$ & $32.8 \%(180)$ & $4.2 \%(14)$ & $0.0 \%(0)$ & $1.4 \%(1)$ & $0.0 \%(0)$ & $0.0 \%(0)$ & $0.0 \%(0)$ & $<0.001$ \\
\hline \multicolumn{9}{|l|}{ LOS } \\
\hline $\mathrm{ICU}^{\mathrm{b}}$ (day) & $4.6(1.4-10.5)$ & $4.7(0.9-15.8)$ & $2.6(0.7-7.8)$ & $6.3(2.3-15.0)$ & $3.2(1.4-7.8)$ & $2.2(0.3-4.1)$ & $7.7(6.6-14.2)$ & 0.135 \\
\hline Hospital ${ }^{b}$ (day) & $11.1(4.6-25.7)$ & $12.3(1.1-37.9)$ & $11.2(0.9-28.8)$ & $14.3(5.2-30.2)$ & $20.6(7.6-27.7)$ & $5.4(0.3-19.4)$ & $43.4(10.2-97.4)$ & 0.037 \\
\hline \multicolumn{9}{|l|}{ Duration } \\
\hline $\begin{array}{c}\text { Episode of } \\
\qquad H^{b}(h)\end{array}$ & 50.6 (24.9-99.3) & $54.5(25.1-87.2)$ & $69.4(36.8-89.5)$ & $71.3(39.0-90.0)$ & $28.4(15.1-77.2)$ & $\begin{array}{l}69.5(28.0- \\
139.0)\end{array}$ & $54.6(35.4-74.0)$ & 0.137 \\
\hline
\end{tabular}

died within $24 \mathrm{~h}$, its 28 -day mortality of $41.4 \%$ ranked fourth among all subgroups. The acute respiratory failure and acute on chronic respiratory failure subgroups had similar survival curves and outcome, with 39.4 and $37.8 \%$ mortality at 28 days. The lowest mortality rate of $33.3 \%$ at
28 days was observed in the hyperthermia subgroup. The survival curves by cause of $\mathrm{HH}$ are presented in Fig. 2 .

The 28-day mortality also increased significantly $(P<0.001)$ with severity of $\mathrm{HH}$, ranging from $33.2 \%$ in the "5-10× ULN" subgroup to $55.4 \%$ in the "> $20 \times$ ULN" 
Table 3 continued

\begin{tabular}{|c|c|c|c|c|c|c|c|c|}
\hline \multirow[t]{2}{*}{ Variable } & \multicolumn{8}{|l|}{ Cause } \\
\hline & $\begin{array}{l}\text { Cardiac failure } \\
(n=548)\end{array}$ & $\begin{array}{l}\text { Septic shock } \\
(n=333)\end{array}$ & $\begin{array}{l}\text { Hypov- } \\
\text { olemic shock } \\
(n=105)\end{array}$ & $\begin{array}{l}\text { Acute res- } \\
\text { piratory failure } \\
(n=71)\end{array}$ & $\begin{array}{l}\text { Acute } \\
\text { on chronic res- } \\
\text { piratory failure } \\
(n=37)\end{array}$ & $\begin{array}{l}\text { Pulmonary } \\
\text { embolism } \\
(n=16)\end{array}$ & $\begin{array}{l}\text { Hyperthermia } \\
(n=6)\end{array}$ & $P$ \\
\hline $\begin{array}{c}\text { Recovery of } \\
H H^{b}(h)\end{array}$ & $33.8(3.4-67.5)$ & $34.3(14.3-60.9)$ & $41.7(21.1-65.1)$ & $31.0(16.2-62.1)$ & $39.9(11.6-89.3)$ & $35.3(10.5-65.4)$ & $29.6(21.7-43.3)$ & 0.810 \\
\hline \multicolumn{9}{|c|}{ 28-day mortality } \\
\hline$I C U^{a}$ & $37.8 \%(207)$ & $48.3 \%(161)$ & $40.0 \%(42)$ & $33.8 \%(24)$ & $27.0 \%(10)$ & $56.2 \%(9)$ & $33.3 \%(2)$ & 0.012 \\
\hline In-hospital ${ }^{a}$ & $41.4 \%(227)$ & $52.9 \%(176)$ & $43.8 \%(46)$ & $39.4 \%(28)$ & $37.8 \%(14)$ & $56.2 \%(9)$ & $33.3 \%(2)$ & 0.029 \\
\hline \multicolumn{9}{|c|}{$\begin{array}{l}\text { APS-II acute physiology II, AST aspartate aminotransferase, ASTmax maximum AST value, } B M I \text { body mass index, } H H \text { hypoxic hepatitis, IABP intra-aortic balloon pump, } \\
\text { ICU intensive care unit, LOS length of stay, LV left ventricle, } P P \text { value, Q1 first quartile, Q3 third quartile, } R V \text { right ventricle, SAPS-II simplified acute physiology II, SOI } \\
\text { severity of illness, T-ASTmax time point of maximum AST value, ULN upper limit of normal }\end{array}$} \\
\hline \multicolumn{9}{|l|}{ a $\%(n)$} \\
\hline \multicolumn{9}{|c|}{ b Median (Q1-Q3) } \\
\hline \multicolumn{9}{|c|}{ c AtT-ASTmax $\pm 12 \mathrm{~h}$} \\
\hline
\end{tabular}

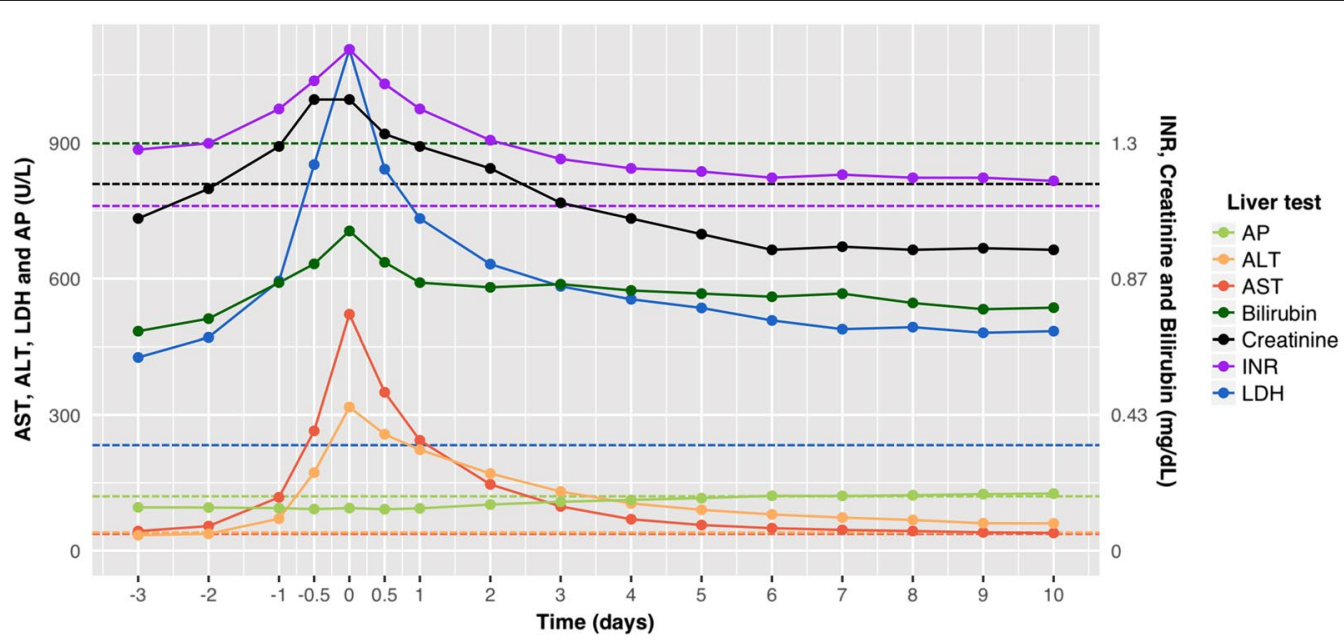

Fig. 3 Time trend of liver tests in hypoxic hepatitis. Time trend of the median AP, ALT, AST, bilirubin, creatinine, INR and LDH levels in hypoxic hepatitis. T-ASTmax is designated as time 0. AST, ALT, LDH and AP levels are measured in U/L and are plotted using the left y-axis. Bilirubin and creatinine levels are measured in $\mathrm{mg} / \mathrm{dL}$ and plotted using the right $\mathrm{y}$-axis. INR has no unit, but is also plotted using the right $y$-axis. AP alkaline phosphatase, ALT alanine aminotransferase, AST aspartate aminotransferase, INR international normalized ratio, LDH lactate dehydrogenase

subgroup. There was no significant difference $(P=0.363)$ in 28-day mortality between men $(39.8 \%)$ and women (42.4\%).

\section{Discussion}

Hypoxic hepatitis is a type of acute hepatic injury in critically ill patients caused by cardiac, circulatory or respiratory failure. While commonly known and referred to as "shock liver" in daily clinical practice, little research has been done. However, early recognition of $\mathrm{HH}$ and management of its underlying cause and complications may improve ultimate outcome. For a long time, only studies with a relatively small number of patients have been conducted. In 2015, a meta-analysis of 24 studies that included 1782 patients summarized the available evidence, but still lacked detailed information on important patient characteristics, biochemical findings and clinical course [2]. To overcome these shortcomings, Aboelsoud et al. recently performed an extensive analysis of the Medical Information Mart for Intensive Care III 

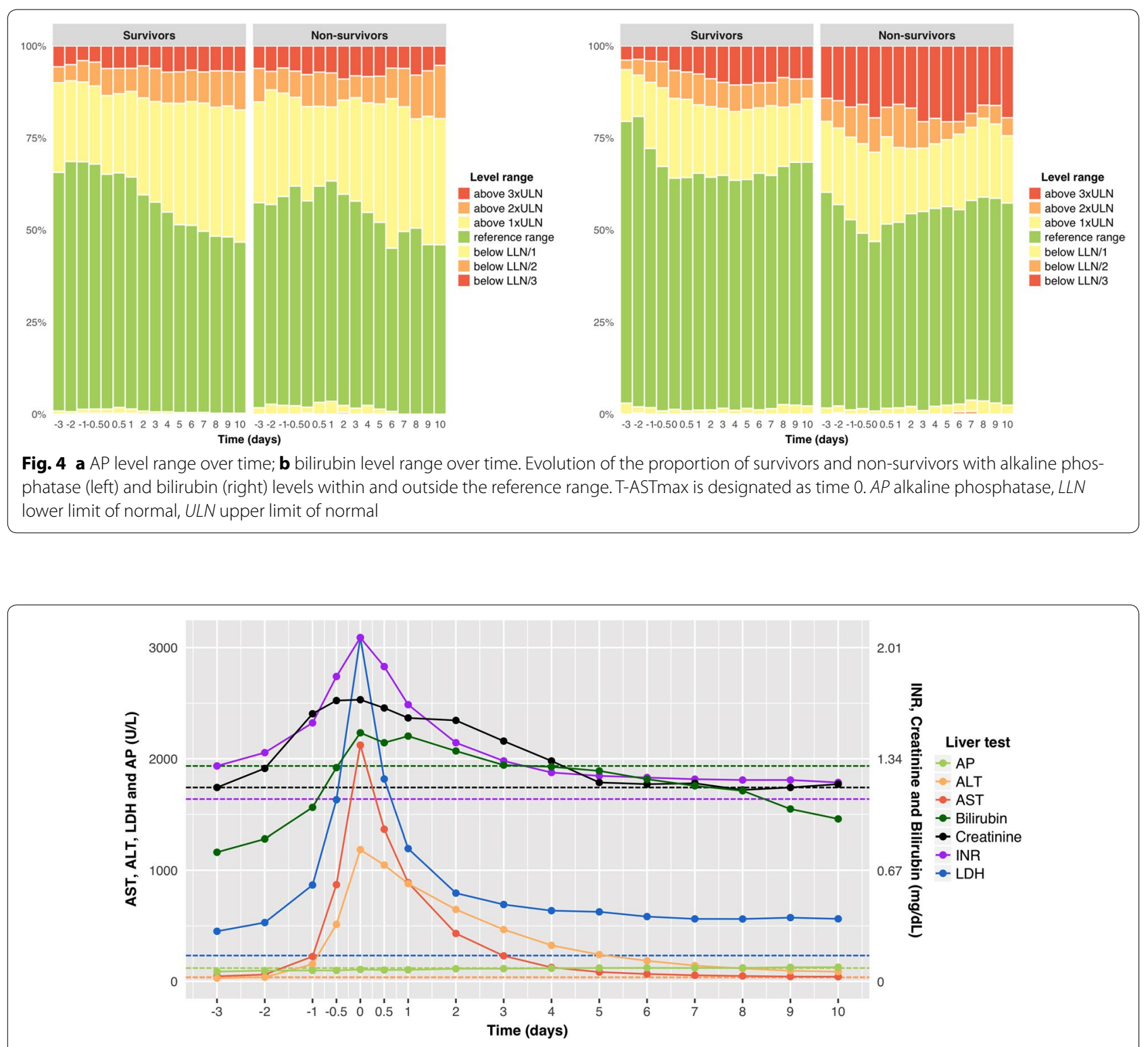

Fig. 5 Time trend of liver tests in hypoxic hepatitis of the ASTmax subgroup 5-10x ULN. Time trend of the median AP, ALT, AST, bilirubin, creatinine, INR and LDH levels in hypoxic hepatitis. T-ASTmax is designated as time 0. AST, ALT, LDH and AP levels are measured in U/L and are plotted using the left $\mathrm{y}$-axis. Bilirubin and creatinine levels are measured in $\mathrm{mg} / \mathrm{dL}$ and plotted using the right $\mathrm{y}$-axis. INR has no unit, but is also plotted using the right y-axis. AP alkaline phosphatase, ALT alanine aminotransferase, AST aspartate aminotransferase, INR international normalized ratio, LDH lactate dehydrogenase

(MIMIC-III) research database. Their study comprised 565 patients with $\mathrm{HH}$ and was the largest cohort study published to date [8].

In our study of 1116 critically ill patients with $\mathrm{HH}$, we investigated in detail its incidence, causes, evolution and outcome. We used an AST cutoff of at least 5 times the ULN (i.e., 155 and $185 \mathrm{U} / \mathrm{L}$ for females and males, respectively) to define $\mathrm{HH}$, while other studies have used higher cutoff values ranging from 400 to $3000 \mathrm{U} / \mathrm{L}$ [2]. HH has been histologically proven to occur even in patients with moderately elevated AST levels (at AST levels of 252 and $300 \mathrm{IU} / \mathrm{L}$ in the cohort of Cohen et al. [13] and Bynum et al. [14], respectively). Additionally, by using a lower cutoff, the sensitivity to identify patients with $\mathrm{HH}$ could be increased. However, this approach could also result in a lower specificity, leading to an increased number of patients that are falsely diagnosed as having $\mathrm{HH}$. In order to maintain a high specificity, we have thoroughly reviewed the clinical notes, the time trend of liver tests and the discharge summaries of the 4012 identified 


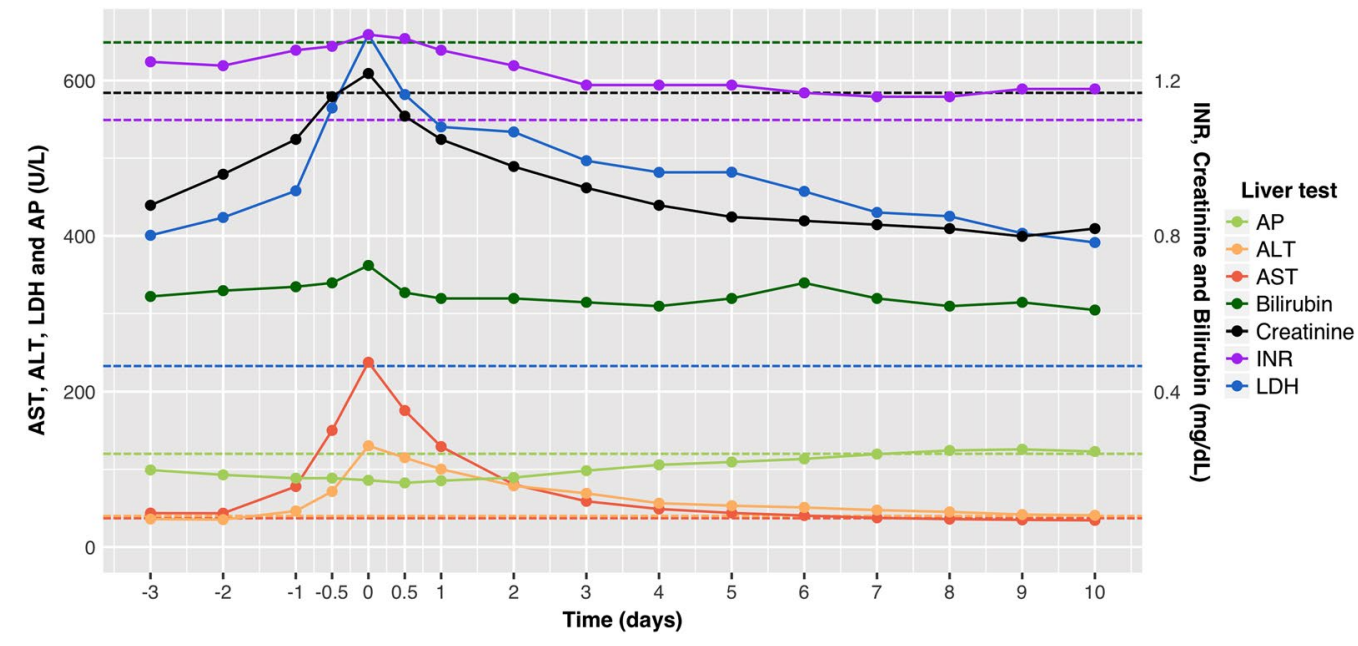

Fig. 6 Time trend of liver tests in hypoxic hepatitis of the ASTmax subgroup 10-20x ULN. Time trend of the median AP, ALT, AST, bilirubin, creatinine, INR and LDH levels in hypoxic hepatitis. T-ASTmax is designated as time 0 . AST, ALT, LDH and AP levels are measured in U/L and are plotted using the left $y$-axis. Bilirubin and creatinine levels are measured in $\mathrm{mg} / \mathrm{dL}$ and plotted using the right $\mathrm{y}$-axis. INR has no unit, but is also plotted using the right y-axis. AP alkaline phosphatase, ALT alanine aminotransferase, AST aspartate aminotransferase, INR international normalized ratio, LDH lactate dehydrogenase

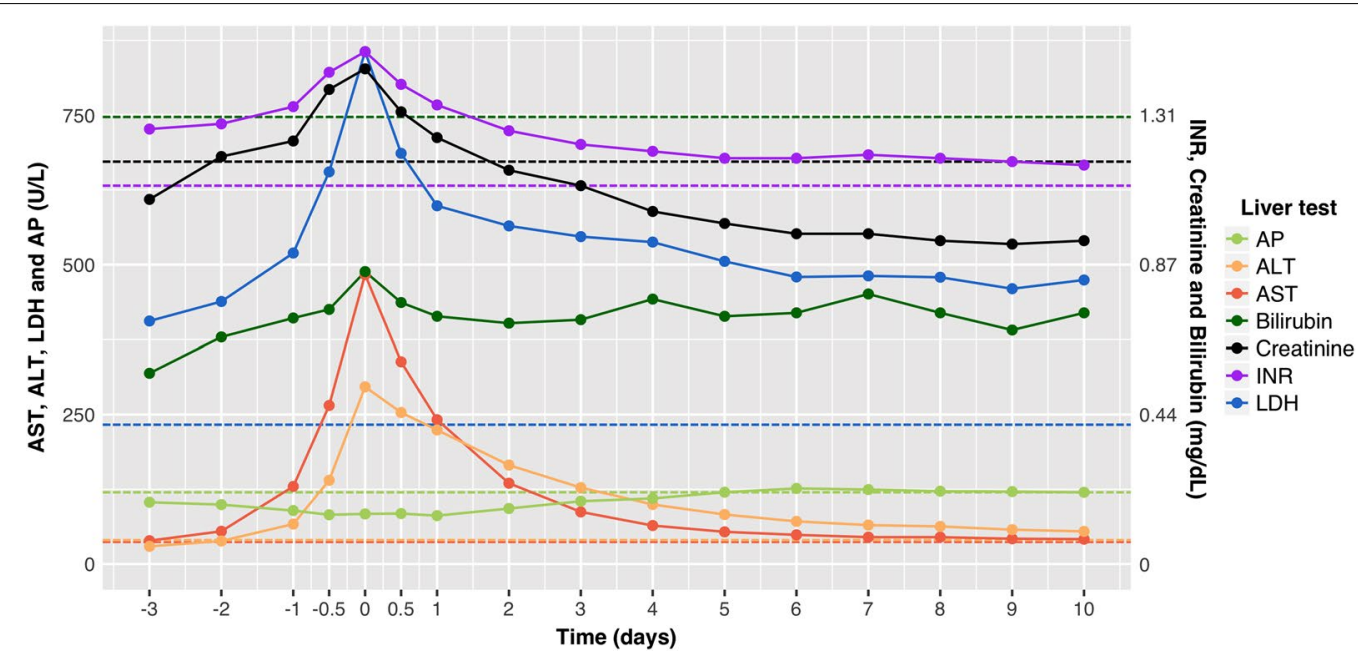

Fig. 7 Time trend of liver tests in hypoxic hepatitis of the ASTmax subgroup > 20x ULN. Time trend of the median AP, ALT, AST, bilirubin, creatinine, INR and LDH levels in hypoxic hepatitis. T-ASTmax is designated as time 0 . AST, ALT, LDH and AP levels are measured in U/L and are plotted using the left $y$-axis. Bilirubin and creatinine levels are measured in $\mathrm{mg} / \mathrm{dL}$ and plotted using the right $\mathrm{y}$-axis. INR has no unit, but is also plotted using the right y-axis. AP alkaline phosphatase, ALT alanine aminotransferase, AST aspartate aminotransferase, INR international normalized ratio, LDH lactate dehydrogenase

patients whose AST level exceeded our cutoff value. Our high number of excluded patients (70.0\%) after applying extensive exclusion criteria may reflect a high specificity. In contrast, Aboelsoud et al. [8] used a cutoff of 800 $\mathrm{U} / \mathrm{L}$ for both AST and ALT to include patients, but only excluded $24.3 \%$ of these patients based on evidence of acetaminophen poisoning, acute viral hepatitis or liver surgery.
Our study uncovered interesting results. Firstly, it indicated that $\mathrm{HH}$ may be more common than previously thought. In our study, an incidence of $4.0 \%$ was observed, which is higher than the pooled incidence of $2.5 \%$ from a meta-analysis of 1782 patients [2] and the incidence of $1.5 \%$ in the MIMIC-III cohort [8]. Moreover, approximately one in five critically ill patients that have died during their ICU stay had developed $\mathrm{HH}$. This 
Table 4 Median laboratory values (Q1-Q3) at different time stages

\begin{tabular}{lcc}
\hline Variable & At start of HH & At T-ASTmax \\
\hline AST $(\mathrm{U} / \mathrm{L})$ & $185.0(155.0-185.0)$ & $521.2(269.1-1581.6)$ \\
ALT $(\mathrm{U} / \mathrm{L})$ & $120.8(70.1-193.5)$ & $317.0(143.5-924.5)$ \\
LDH $(\mathrm{U} / \mathrm{L})$ & $691.8(465.8-1025.7)$ & $1106.4(624.8-2602.8)$ \\
Bilirubin $(\mathrm{mg} / \mathrm{dL})$ & $0.8(0.5-1.6)$ & $1.0(0.6-2.0)$ \\
GGT $(\mathrm{U} / \mathrm{L})$ & $73.1(36.2-160.4)$ & $82.5(41.7-167.0)$ \\
AP $(\mathrm{U} / \mathrm{L})$ & $88.2(61.8-150.9)$ & $94.0(62.9-167.0)$ \\
Lipase $(\mathrm{U} / \mathrm{L})$ & $32.4(18.9-58.9)$ & $33.5(18.5-66.4)$ \\
INR & $1.4(1.2-1.8)$ & $1.6(1.3-2.2)$ \\
PC $\left(10^{3} / \mu \mathrm{L}\right)$ & $165.7(103.6-241.0)$ & $146.0(79.5-218.0)$ \\
WBC $\left(10^{3} / \mu \mathrm{L}\right)$ & $12.5(8.8-17.6)$ & $13.2(9.0-18.5)$ \\
Hb $(\mathrm{g} / \mathrm{dL})$ & $10.1(8.8-12.0)$ & $9.8(8.6-11.5)$ \\
CK $(\mathrm{U} / \mathrm{L})$ & $214.0(77.2-747.2)$ & $322.2(105.1-1102.9)$ \\
Creatinine $(\mathrm{mg} / \mathrm{dL})$ & $1.4(0.9-2.1)$ & $1.4(0.9-2.2)$ \\
Urea $(\mathrm{mg} / \mathrm{dL})$ & $57.1(38.9-91.3)$ & $61.6(40.6-96.4)$ \\
Lactate $(\mathrm{mg} / \mathrm{dL})$ & $31.3(16.2-62.2)$ & $27.4(14.7-56.2)$ \\
\hline
\end{tabular}

$A P$ alkaline phosphatase, $A L T$ alanine aminotransferase, $A S T$ aspartate aminotransferase, $C K$ creatine kinase, GGT gamma-glutamyl transpeptidase, $H b$ hemoglobin, INR international normalized ratio, $L D H$ lactate dehydrogenase, $P C$ platelet count, WBC white blood cell count

higher incidence observed in our study is of course a direct consequence of the lower AST cutoff used. However, it should be noted that the underlying conditions, the typical liver test pattern and the considerable mortality of $33.2 \%$ at 28 days in patients with a less pronounced rise of AST (peak AST level between 5 and 10 times the ULN) are just as much compatible with the diagnosis of $\mathrm{HH}$ as in patients with higher peak AST level. Any sudden increase in aminotransferases above 5 times the ULN should therefore prompt the clinician to actively search for any changes in physiological parameters and maintain strict hemodynamic and respiratory control.

Secondly, the typical liver test pattern that suggests diagnosis of $\mathrm{HH}$ was observed in all subgroups of our study cohort. HH was characterized by (1) a sudden and significant increase of aminotransferases with an AST/ ALT ratio greater than 1, (2) a similar rise in LDH mostly exceeding both AST and ALT and (3) a steady decline of aminotransferases with reversal of the AST/ALT ratio approximately 1.5 days after T-ASTmax. In survivors, LDH normalized first, followed by AST and finally ALT (median of 5.1, 6.4 and 8.6 days after T-ASTmax, respectively). Bilirubin followed a similar pattern as the aminotransferases, but the magnitude of its increase was much less pronounced, with median levels never exceeding the ULN. The associated prolongation of INR and impairment of renal function as described by Raurich et al. [6] were also confirmed in this study.

Finally, we observed a 28 -day mortality of $45.0 \%$, which is in line with the reported mortality of $49 \%$ from a meta-analysis of 1782 patients [2] and the mortality of $44.1 \%$ in the MIMIC-III cohort [8]. HH was especially associated with high mortality rates early in its course, with roughly $40 \%$ of deaths occurring within $24 \mathrm{~h}$ after T-ASTmax. This was mainly driven by the severity of the underlying causes [2], although the ultimate cause of death was not inferred in this study. The magnitude of AST increase also appeared to be correlated with mortality on univariate analysis.

Our study has a number of strengths. To our knowledge, it is by far the largest cohort study of patients with $\mathrm{HH}(n=1116)$. It comprises twice as many patients as the second largest cohort study, which was recently published and included 565 patients [8]. Additionally, by using a low AST cutoff of 5 times the ULN while simultaneously excluding many conditions that could mimic $\mathrm{HH}$, we are confident that we could identify nearly all patients with $\mathrm{HH}$ at our center with a low risk of misdiagnosis. Our study may have some limitations inherent in its retrospective design. However, we believe that the retrospective design had little to no impact on our results given the entirely computerized monitoring and management at our ICU, the detailed review of patient records and the high number of patients included. Nevertheless, we should be careful to generalize these results to a broader patient population since only critically ill patients older than 18 years and only from a single university hospital ICU were included.

\section{Conclusions}

This study surpasses by far the largest cohort of critically ill patients with hypoxic hepatitis. $\mathrm{HH}$ is more common than previously thought with an ICU incidence of $4.0 \%$, and it is associated with a high all-cause mortality of $45.0 \%$ at 28 days. The main causes of $\mathrm{HH}$ are cardiac failure and septic shock, which include more than $3 / 4$ of all episodes. Clinicians should search actively for any underlying hemodynamic or respiratory instability even in patients with moderately increased AST levels.

\section{Abbreviations}

$\mathrm{HH}$ : hypoxic hepatitis; AST: aspartate aminotransferase; ICU: intensive care unit; ALT: alanine aminotransferase; LDH: lactate dehydrogenase; ULN: upper limit of normal; CK: creatine kinase; AP: alkaline phosphatase; INR: international normalized ratio; BMI: body mass index; APS-II: acute physiology II; SAPS-II: simplified acute physiology II; T-ASTmax: time point of maximum AST value; ASTmax: maximum AST value; Q1: first quartile; Q3: third quartile; MIMIC-III: Medical Information Mart for Intensive Care III.

\section{Authors' contributions}

AV and LV designed the study, collected data, performed and interpreted statistical analysis, and drafted the manuscript. Both authors contributed equally to this study and are the joint first authors. AD performed and interpreted statistical analysis, and drafted the manuscript. KC, HV and DB designed the study and revised the manuscript. JD designed the study, interpreted 
statistical analysis and revised the manuscript. All authors read and approved the final manuscript.

\section{Author details}

${ }^{1}$ Faculty of Medicine and Health Sciences, Ghent University, Ghent, Belgium.

${ }^{2}$ Department of Internal Medicine, Ghent University Hospital, Ghent, Belgium.

${ }^{3}$ Department of Intensive Care Medicine, Ghent University Hospital, De PinteIaan 185, 9000 Ghent, Belgium. ${ }^{4}$ Department of Hepatology and Gastro-Enterology, Ghent University Hospital, Ghent, Belgium.

\section{Acknowledgements}

Not applicable.

\section{Competing interests}

The authors declare that they have no competing interests.

\section{Availability of data and materials}

The datasets used and analyzed during the current study are available from the corresponding author on reasonable request.

\section{Consent for publication}

Not applicable.

\section{Ethics approval and consent to participate}

In accordance with the ethical standards of our hospital's Institutional Ethics Committee (Project Numbers 2015/0796-0797), informed consent was not necessary for analyses of demographic, physiological and hospital-outcome data, because this retrospective observational study did not modify existing diagnostic or therapeutic strategies.

\section{Funding}

None.

\section{Publisher's Note}

Springer Nature remains neutral with regard to jurisdictional claims in published maps and institutional affiliations.

\section{Appendix}

Received: 6 July 2017 Accepted: 1 January 2018

Published online: 30 January 2018
References

1. Henrion J. Hypoxic hepatitis. Liver Int. 2012;32(7):1039-52.

2. Tapper EB, Sengupta N, Bonder A. The incidence and outcomes of ischemic hepatitis: a systematic review with meta-analysis. Am J Med. 2015;128(12):1314-21.

3. Henrion J, Schapira M, Luwaert R, Colin L, Delannoy A, Heller FR. Hypoxic hepatitis: clinical and hemodynamic study in 142 consecutive cases. Medicine (Baltimore). 2003;82(6):392-406.

4. Birrer R, Takuda Y, Takara T. Hypoxic hepatopathy: pathophysiology and prognosis. Intern Med. 2007;46(14):1063-70.

5. Fuhrmann V, Kneidinger N, Herkner H, Heinz G, Nikfardjam M, Bojic A, et al. Hypoxic hepatitis: underlying conditions and risk factors for mortality in critically ill patients. Intensive Care Med. 2009;35(8):1397-405.

6. Raurich JM, Llompart-Pou JA, Ferreruela M, Colomar A, Molina M, Royo C, et al. Hypoxic hepatitis in critically ill patients: incidence, etiology and risk factors for mortality. J Anesth. 2011;25(1):50-6.

7. Fuchs S, Bogomolski-Yahalom V, Paltiel O, Ackerman Z. Ischemic hepatitis: clinical and laboratory observations of 34 patients. J Clin Gastroenterol. 1998;26(3):183-6.

8. Aboelsoud MM, Javaid Al, Al-Qadi MO, Lewis JH. Hypoxic hepatitis_its biochemical profile, causes and risk factors of mortality in critically-ill patients: a cohort study of 565 patients. J Crit Care. 2017:41:9-15.

9. Dunn GD, Hayes P, Breen KJ, Schenker S. The liver in congestive heart failure: a review. Am J Med Sci. 1973;265(3):174-89.

10. Ebert EC. Hypoxic liver injury. Mayo Clin Proc. 2006;81(9):1232-6.

11. Whitehead MW, Hawkes ND, Hainsworth I, Kingham JG. A prospective study of the causes of notably raised aspartate aminotransferase of liver origin. Gut. 1999;45(1):129-33.

12. Johnson RD, O'Connor ML, Kerr RM. Extreme serum elevations of aspartate aminotransferase. Am J Gastroenterol. 1995;90(8):1244-5.

13. Bynum TE, Boitnott JK, Maddrey WC. Ischemic hepatitis. Dig Dis Sci. 1979;24(2):129-35.

14. Cohen JA, Kaplan MM. Left-sided heart failure presenting as hepatitis. Gastroenterology. 1978;74(3):583-7.

15. Core Team R. R: a language and environment for statistical computing. Vienna: R Foundation for Statistical Computing; 2016.

\section{Submit your manuscript to a SpringerOpen ${ }^{\circ}$ journal and benefit from:}

- Convenient online submission

- Rigorous peer review

- Open access: articles freely available online

- High visibility within the field

- Retaining the copyright to your article

Submit your next manuscript at $\boldsymbol{\nabla}$ springeropen.com 